\title{
DESIGN OF ANALYTICAL MODEL FOR ULTRA WIDEBAND SYSTEM
}

\author{
Alpana P. Adsul ${ }^{1}$ and Dr. Shrikant K. Bodhe ${ }^{2}$ Member IEEE. \\ ${ }^{1}$ Department of Computer Engineering,Vishwakarma Institute of Technology, \\ Pune-37, India \\ alpana.adsul@gmail.com, alpanaprashant@yahoo.co.in \\ ${ }^{2}$ Director, Bosh Technologies, Pune-37, India \\ s_k_bodhe@yahoo.co.in
}

\begin{abstract}
The UWB (Ultra Wideband) technology has drawn phenomenal interest in industry as well as academia. Ultra Wide Band impulse radios are microwave systems that communicate using baseband pulses of very short duration. UWB systems transmit information data over a wide frequency spectrum with low power consumption and high speed for local area wireless network applications. Unlike the traditional digital communication method based on a carrier wave, UWB is pulse based. Pulse Generation, modulation, and multiple access are time domain dependent functions. This paper presents the development of analytical model for UWB system. A theoretical reference for UWB system performances is designed in non-ideal channels. In this mathematical models for biphase, pulse-position and hybrid modulation are developed. The detection rules are formulated for detecting signals in AWGN channels. The performance of UWB system is described with the help of BER. The BER of a UWB system depends on the modulation scheme and detection method it uses. It is observed that for optimum performance modulation parameter selection is important.
\end{abstract}

\section{KEYWORDS}

$U W B, A W G N, P P M, T M-U W B$

\section{INTRODUCTION}

The world of ultra-wideband (UWB) has changed dramatically in very recent history. In the past 20 years, UWB was used for radar, sensing, military communications and niche applications. A substantial change occurred in February 2002, when the FCC issued a ruling that UWB could be used for data communications as well as for radar and safety applications. The proliferation of consumer electronics devices, both in the home and the office, is driving a need for robust and high speed wireless networks. There is a very strong case for short-range wireless networks, which operate only over a few feet and provide data rates in the hundreds of Mbps. The band allocated to UWB communications is a staggering $7.5 \mathrm{GHz}$, by far the largest allocation of bandwidth to any commercial terrestrial system. UWB impulse radio systems transmit signals with a bandwidth larger than $500 \mathrm{MHz}$ or a center frequency to bandwidth ratio more than $20 \%$. The UWB transmission has been enabled by the Federal Communication Commission (FCC) which allowed the reuse of the pre-existing bands from 3.1 to $10.6 \mathrm{GHz}$ by $\mathrm{UWB}$ devices.

There are two different FCC masks, one for indoor environments and another for outdoor ones. The main difference between them resides in the power limit of the UWB signal. This spread UWB spectrum can be used in two different ways: with single-band and multi-band approaches. In the former, the signal is transmitted using the full spectrum; this kind of transmission is known as carrier-less or pulse-based UWB. In the latter, the signal occupies a relatively small 
fraction of the spectrum because the whole spectrum is divided in several sub-bands; this kind of transmission is also known as carrier-UWB. In multiband UWBs, for allowing the use of these sub-bands and improve the Bit-Error-Rate, techniques like Orthogonal Frequency Domain Multiplexing (OFDM) are employed.

This novel technology has recently received much attention for major advances in wireless applications such as wireless communication networking, radar, imaging, and positioning systems. It is fairly common for an average home to have a few digital cameras, a few portable music players and one or more portable digital assistants, in addition to cellular phones and external hard drives. As the number of devices in a home grows, so does the number of wires connecting these peripherals together. Ultra-wideband technology brings the convenience and mobility of wireless communications to high-speed interconnects in devices throughout the digital home and office. Designed for short-range wireless personal area networks (WPANs), UWB is an emerging technology for freeing people from wires, enabling wireless connection of multiple devices for transmission of video audio, and other high-bandwidth data.

UWB systems show a number of important advantages:

- accurate position location and ranging, due to the fine time resolution;

- no significant multipath fading due to fine time resolution;

- multiple access due to wide transmission bandwidths;

- possibility of extremely high data rates;

- covert communications due to low transmission power operation; and

- possible easier material penetration due to the presence of components at different frequencies.

UWB differs substantially from conventional narrowband radio frequency (RF) and spread spectrum technologies (SS), such as Bluetooth Technology and IEEE $802.11 \mathrm{a} / \mathrm{b} / \mathrm{g}$. The UltraWide-Band (UWB) technology allows communication using pulses of a short duration which have a bandwidth occupation of several GHz. The main differences from the Narrow-Band communication systems are relevant: in fact, they use a short bandwidth and the transmission process is done by modulating a particular signal known as carrier. For example in a Wi-Fi environment data is transmitted in a band of few $\mathrm{MHz}$ with a carrier of $2.54 \mathrm{GHz}$. Instead, the nature of the UWB signal allows the reuse of the typical Narrow-Band transmission frequencies, allowing elevate bit rates and high multipath resolutions. The applications of this technology go from Multimedia to Internet connectivity, and its bit-rates are within a hundred Kbps and few Mbps.

One of the enormous potentials of UWB, however, is the ability to move between the very high data rate, short link distance and the very low data rate, longer link distance applications. The trade-off is facilitated by the physical layer signal structure. The very low transmit power available invariably means multiple, low energy, UWB pulses must be combined to carry 1 bit of information. When UWB signals are applied in communications systems, some unique advantages such as higher processing gain and multipath resolution, deeper material penetration and more covert operation could be offered over conventional narrowband systems $[1,2]$. For low complexity implementation, UWB systems usually use time-hopping impulse train with pulse position modulation to carry data information.

There are different schemes of UWB Systems, such as OFDM, or impulse radio UWB. Transmitted Reference UWB uses ultra-short information bearing pulses and thus promises high speed, high precision, resolved multipath and simpler receiver structures. TR-UWB uses an ultra-short reference pulse followed by the actual information bearing pulse to transmit data. These pairs of pulses are called doublets. The reference pulse and the information bearing pulse 
are distorted by the same channel. At the receiver, the pulses are correlated and integrated, allowing the receiver to gather the whole channel energy.

\section{RELATED WORK.}

Impulse radio communication systems and impulse radars both utilize very short pulses in transmission that results in an ultra-wideband spectrum. For radio applications, this communication method is also classified as a pulse modulation technique because the data modulation is introduced by pulse position modulation (PPM). Time-modulated (TM) impulse radio signal is seen as a carrier-less baseband transmission. The absence of carrier frequency is the very fundamental character that differentiates impulse radio and impulse radar transmissions from narrow-band applications and from direct sequence (DS) spread spectrum (SS) multicarrier (MC) transmissions, which can also be characterized as an (ultra) wideband technique. Time-modulated ultra wideband (TM-UWB) communication is based on discontinuous emission of very short Gaussian pulses or other types of pulse waveforms (monocycles). Each pulse has the ultra wide spectral requirement in frequency domain. This type of transmission does not require the use of additional carrier modulation, as the pulse will propagate well in the radio channel. The technique is therefore a baseband signal approach. This radio concept is referred as impulse radio (IR).

In literature, there are different solutions for UWB devices that is there are two main kinds of receivers in UWB scenario: the coherent and the non-coherent ones. The former is the optimal receiver for signals transmitted over an Additive-White-Gaussian-Noise (AWGN) channel. It performs the correlation with the channel estimation. This waveform should be the same as that of the received signal. Such a signal is very difficult to generate in practice because the transmitted pulse is distorted by the antennas and by the channel. The need to make the template waveform the same as that of the received signal also makes the receiver more complex. One way to avoid this complexity is to approximate the template waveform by using the transmitter pulse. It is also possible to ignore the template waveform and to rely on the pulse shaping caused by the finite bandwidth of the transmitting and receiving antennas. While coherent receivers acquire pulses by correlating the received pulse with channel estimation, the noncoherent receivers does not consider any information on the shape of the pulse and does not perform any correlation. They demodulate data and perform the other phases which characterize data reception taking into account e.g. the pulse energy; the main drawback of this approach regards the lowering of the Bit-Error-Rate (BER) given a Signal-to-Noise Ratio with respect to coherent receivers.

Considerable experiments on the characterization of UWB signal propagation channel [1-3] and efforts on the performance evaluation of UWB systems [4-8] have been made over the recent years. In multiple access environments, the performance evaluation was normally carried out through union bound approximation or numerical simulation [5, 8]. However, even for single user UWB system, the exact formula of the bit error performance cannot be readily found in the literature. In this paper, the demodulation methods of three impulse train modulation schemes $[9,11]$, namely, biphase modulation (BPM), pulse position modulation (PPM) and hybrid modulation which combines BPM and PPM together, in additive white Gaussian noise (AWGN) channel are investigated. The exact bit error probabilities of UWB systems using these modulation schemes are derived. The results can be used as theoretical references for evaluating system performances in other non-ideal multipath and/or multiple access environments.

Many of the approaches in the UWB literature adopt coherent correlation receivers [11], [12], [13], which demodulate the received signal by correlating it, with a well-designed template signal. The correlation receiver is optimal over AWGN and non-ISI multipath channels in the sense of minimizing the probability of data detection error [14], [15]. However, it has to face great design challenges [16]. In order to correlate the received signal with the template, the receiver needs to achieve synchronization with inaccuracy much smaller than one pulse duration 
(e.g., a few tens of picoseconds) [17]-[18]. The correlation receiver also requires precise template signal design, which is difficult due to linear and nonlinear distortions of the signal during its transmission [12].A simplified realization of the correlation receiver is the rake receiver [14], [19]-[20]. However, the rake receiver also suffers from synchronization and channel estimation problems, and requires a large number of branches to capture enough signal energy, which results in a very high implementation complexity. Non-coherent UWB receivers such as the autocorrelation receiver and the energy detector are promising alternatives to coherent receivers. Transmitted reference (TR) and differential (DF) schemes are two primary approaches in the literature employing autocorrelation receivers. They both take the received signal as the template, circumventing problems of channel estimation and multipath energy combining. A unique advantage of UWB autocorrelation receivers is their robustness to synchronization errors, which has not been explored yet to date. In coherent receivers, accurate synchronization is absolutely needed to align the received signal with the template signal. Even a small synchronization error leads to great performance degradations. However, in noncoherent UWB receivers, synchronization information is needed only for deciding the integration region of the autocorrelator and the sampling point at the integrator output. Due to the low pulse duty-cycle and the multi-frame structure of the UWB signal, inaccurate selection of the integration region and the sampling point due to synchronization errors may not result in a great performance degradation, which means that noncoherent UWB receivers are robust to synchronization errors.

The rest of the paper is organized as follows. In Section II, the mathematical models for describing impulse train modulated UWB signals in general long sequence case are developed and the decision rules for optimum detection of the modulated signals over ideal AWGN channel are proposed. Section IV illustrates how to select the time shift parameters in PPM and hybrid modulation to optimize the system performance. Finally, conclusions are drawn in Section V.

\section{Mathematical Model}

We consider UWB signals generated by modulating a pseudorandom (PN) impulse train with input data information and an ideal transmission channel with only AWGN interference. The simplified transmission model is shown in Figure 1. In this model, $c(t)$ denotes the waveform of the PN impulse train. $x(t)$ is the modulated signal that is then directly applied to a transmitter antenna with impulse response $w_{t}(t)$ for radiation. The receiver antenna receives the transmitted UWB signal and changes the shape of $w_{t}(t)$ to $w_{r}(t) . y(t)$ and $z(t)$ represent respectively the signal and interference components at the output of the receiver antenna. The attenuation factor $\alpha$ models the propagation of the UWB signal over the channel.

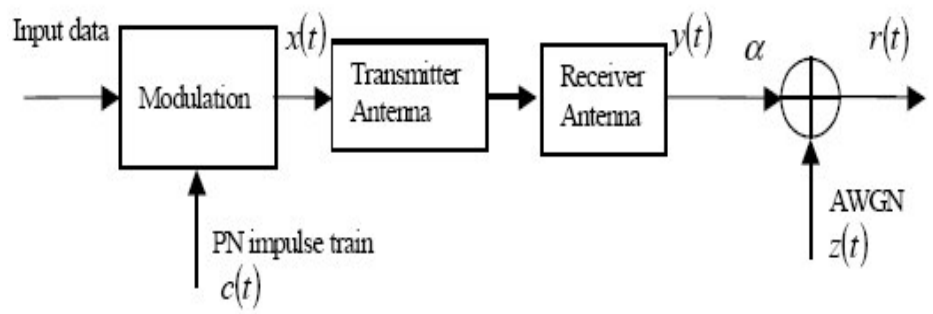

Figure 1: Basic UWB Transmission Model.

The received UWB signal can be expressed as 
$r(t)=\alpha y(t)+z(t)$

where $y(t)=\int_{-\infty}^{+\infty} x(\tau) w_{r}(t-\tau) d \tau$.

The waveform of the PN impulse train can be generally expressed as

$$
c(t)=\sum_{i=-\infty}^{+\infty} c_{i} \delta\left(t-i T_{i}-d_{i}\right),
$$

Where $\delta(t)$ denotes Dirac delta function, $T_{c}$ is the nominal impulse repetition period (called chip time), $\left\{c_{i}\right\}$ is a PN binary sequence of period $N_{p}$ (called direct sequence), $d_{i} \in\left[0, T_{c}\right)$ is a PN time delay associated with each impulse and the sequence $\left\{d_{i}\right\}$ (called time-hopping sequence) is also periodic with period $N_{p}$. We further assume that long direct sequence and time-hopping sequence are used, i.e., the period of the PN impulse train $T_{p}$ is longer than the data symbol duration $T_{s}$, and the ratio of $T_{s}$ over $T_{p}$ is rational, i.e., $T / T_{p}=K / L$, where $L$ and $K$ are relatively prime integers. This implies that $L$ data symbols take the same time duration as $K$ periods of the PN impulse train. By defining

$c^{l}(t)=\left\{\begin{array}{lc}c\left(t+l T_{s}\right) & \text { for } 0 \leq t<T_{s} \\ 0 & \text { elsewhere }\end{array}\right.$

$c(t)$ can be expressed as

$$
c(t)=\sum_{n=-\infty}^{+\infty} c^{\left((n)_{L}\right)}\left(t-n T_{s}\right)
$$

Where the notation $(n)_{L}$ denotes $n$ modulo $L$.

Now let us consider three different impulse modulation schemes, i.e., BPM which is also called direct sequence modulation, PPM, and the quaternary hybrid modulation. Suppose that for BPM and PPM systems the input data bit at time $n T_{s}$ is $a_{n} \in[-1,+1]$ and for hybrid system the input data symbol at time $n T_{s}$ is $b_{n}=2 a_{n}^{(1)}+a_{n}^{(0)} \in[-3,-1,+1,+3]$ where $a_{n}^{(1)} \in[-1,+1]$ is the most significant bit (MSB) and $a_{n}^{(0)} \in[-1,+1]$ is the least significant bit (LSB). Then the modulated signal and the received noise-free signal can be expressed as

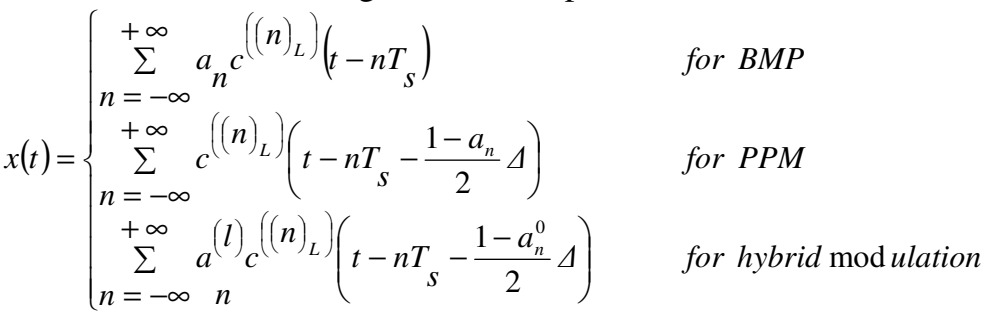

$$
\begin{aligned}
& y(t)= \begin{cases}\sum_{n=-\infty}^{+\infty} a_{n} c^{\left((n)_{L}\right)}\left(t-n T_{S}\right) & \text { for BMP } \\
& \text { for PPM } \\
\sum_{n=-\infty}^{+\infty} y^{\left((n)_{L}\right)}\left(t-n T_{S}-\frac{1-a_{n}}{2} \Delta\right) & \text { for hybrid mod ulation } \\
\sum_{n=-\infty}^{+\infty} a^{(l)} y\left((n)_{L}\right)\left(t-n T_{S}-\frac{1-a_{n}^{0}}{2} \Delta\right) & \end{cases} \\
& r(t)=\alpha y^{\left((n)_{L}\right)}\left(t-n T_{s}\right)+z(t)
\end{aligned}
$$


respectively, where $\Delta$ denotes the time shift when the binary data bit $a_{n}$ (for PPM ) or $a_{n}^{(0)}$ (for hybrid modulation) is -1 and

$$
y^{(l)}(t)=\int_{-\infty}^{+\infty} c^{(l)}(\tau) w_{r}(t-\tau) d \tau
$$

Referring to Equation (1) and assuming that $z(t)$ is Gaussian and has double-sided power spectral density $1 / 2 N_{0}$, the optimum detection of the transmitted data symbols can be realized using maximum-likelihood criterion. Defining two decision variables

$$
U=\alpha \int_{-\infty}^{+\infty} r\left(t+n T_{s}\right) y^{\left((n)_{L}\right)}(t) d t
$$

and

$$
V=\alpha \int_{-\infty}^{+\infty} r\left(t+n T_{s}\right) y^{\left((n)_{L}\right)}(t-\Delta) d t
$$

The decision rule for biphase modulated UWB signal is

$$
a_{n}= \begin{cases}+1 & \text { if } U \geq 0 \\ -1 & \text { if } U<0\end{cases}
$$

which represents coherent receiving because the knowledge of the sign of $\alpha$ is required. For pulse position modulated UWB signal, the coherent receiving decision rule is

$$
a_{n}= \begin{cases}+1 & \text { if } U \geq V \\ -1 & \text { if } U<V\end{cases}
$$

and the noncoherent energy detection decision rule is

$$
a_{n}= \begin{cases}+1 & \text { if }|U| \geq|V| \\ -1 & \text { if }|U|<|V|\end{cases}
$$

Finally, the decision rules for hybrid modulated UWB signals are

$$
a_{n}^{(1)}= \begin{cases}+1 & \text { if } U \geq|V| \quad \text { or } V \geq|U| \\ -1 & \text { if } U<-|V| \text { or } V<-|U|\end{cases}
$$

and

$$
a_{n}^{(0)}= \begin{cases}+1 & \text { if }|U| \geq|V| \\ -1 & \text { if }|U|<|V|\end{cases}
$$

\section{Bit ERror ANALYSIS}

Let us consider the biphase modulated UWB System. According to Equation (1) and (6) and assuming that $a_{n}= \pm 1$ is transmitted at time $t=n T_{s}$, the received UWB signal is $r(t)=\alpha y^{\left((n)_{L}\right)}\left(t-n T_{s}\right)+z(t)$

Substituting it into (7), the decision variable can be expressed as $U=E_{b}+Z$,

Where $E_{b}=\alpha^{2} \int_{-\infty}^{+\infty}\left[y^{((n) L)}(t)\right]^{2} d t$ represents the average received signal energy per bit (assume that the energy of the signal $y^{(t)}(t)$ is the same for all $0 \leq l \leq L$ ) and 
$Z=\alpha \int_{-\infty}^{+\infty} z\left(t+n T_{s}\right) y^{((n) L)}(t) d t$ is a Gaussian random variable with zero mean and variance $\sigma^{2}=E\left\lfloor Z^{2}\right\rfloor=\frac{1}{2} N_{0} E_{b}$. Therefore, the probability density function of $U$ is $p(u)=\frac{1}{\sqrt{\pi N_{0} E_{b}}} e^{-\left(u-E_{b}\right)^{2} / N_{0} E_{b}}$

According to decision rule (9), the bit error probability is $P(U<0)=\int_{-\infty}^{0} p(u) d u$ and can be expressed as a function of the normalized signal-to-noise ratio (SNR) $\gamma_{b}=\frac{E_{b}}{N_{0}}$ after some manipulation, i.e.

$$
P_{e}\left(\gamma_{b}\right)=Q\left(\sqrt{2 \gamma_{b}}\right)
$$

where the Q-function is defined by $Q(x)=\frac{1}{\sqrt{2 \pi}} \int_{x}^{+\infty} e^{-t^{2} / 2} d t$.

Note that (17) is the best performance a binary modulation scheme could offer.

For pulse position modulated UWB system, the other decision variable $V$ can be expressed as

$V=E_{b} \rho+Z_{\Delta}$

Where

$$
\rho=\int_{-\infty}^{+\infty} y^{\left((n)_{L}\right)}(t) y^{\left((n)_{L}\right)}(t-\Delta) d t / \int_{-\infty}^{+\infty}\left[y^{\left((n)_{L}\right)}\right]^{2} d t
$$

is the normalized auto-correlation coefficient of $y^{\left((n)_{L}\right)}(t)$ at offset $\Delta$ and $Z_{\Delta}=\alpha \int_{-\infty}^{+\infty} z\left(t+n T_{s}\right) y^{\left((n)_{L}\right)}(t-\Delta) d t$ is a Gaussian random variable with zero mean and variance $\sigma^{2}$. Note that the joint central moment of the two Gaussian variables $U$ and $V$ can be evaluated as

$\mu=E\left[\left(U-E_{b}\right)\left(V-E_{b}\right) \rho\right]=E\left[Z Z_{\Delta}\right]=\frac{1}{2} N_{0} E_{b} \rho$,

which means that $U$ and $V$ are correlated if $\rho \neq 0$. The joint probability density function of them can be expressed as [14]

$$
p(u, v)=\frac{1}{\pi N_{0} E_{b} \sqrt{1-\rho^{2}}} e^{\left(u-E_{b}\right)^{2}-2 \rho\left(u-E_{b}\right)\left(v-E_{b} \rho\right)+\left(v-E_{b}\right)},
$$


According to the decision rule for coherent receiving (10), the probability for correct decision can be evaluated as $\mathrm{P}(U \geq V)=\int^{+\infty} \int^{u} \rho(u, v) d v d u$. The bit error probability, as a function $-\infty-\infty$

of $\gamma_{b}$, can be then derived as

$$
P_{e}\left(\gamma_{b}\right)=1-\int_{-\infty-\infty}^{+\infty} \int_{-\infty}^{u} \rho(u, v) d v d u=Q\left(\sqrt{(1-\rho)} \gamma_{b}\right)
$$

Fig. 2 illustrates this error probability for different values of $\rho$. It is minimized when $\rho=-1$ and becomes $\frac{1}{2}$ when $\rho=1$. For energy detection, according to the decision rule (11),

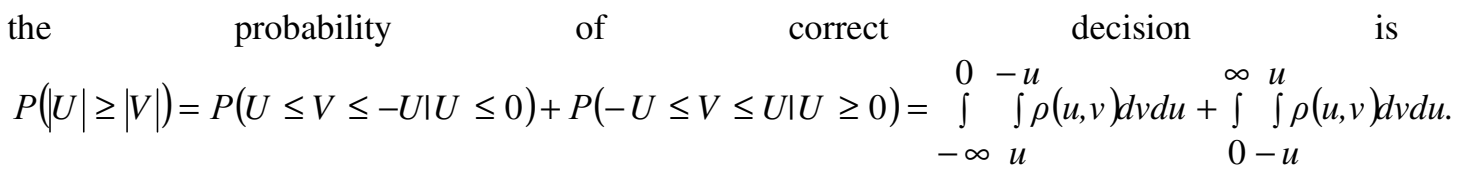

Therefore, the bit error probability is

$P_{e}\left(\gamma_{b}\right)=1-\left[\int_{-\infty}^{0} \int_{u}^{-u} \rho(u, v) d v d u+\int_{0}^{\infty} \int_{-u}^{u} \rho(u, v) d v d u\right]$

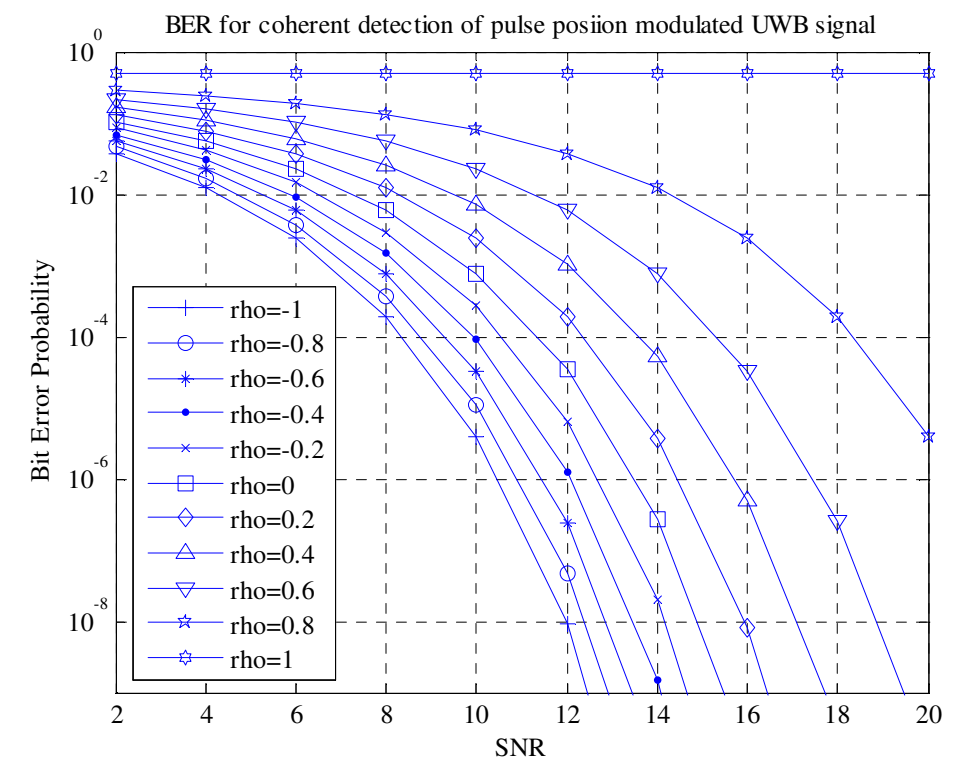

Figure 2. The error probability for coherent detection of pulse position modulated UWB signal corresponding to different value of $\rho$. 


$$
\begin{aligned}
& =Q\left(\sqrt{(1+\rho) \gamma_{b}}\right)+Q\left(\sqrt{(1-\rho) \gamma_{b}}\right) \\
& -2\left\{Q\left(2 \gamma_{b}\right)-\frac{1}{\sqrt{2 \Pi}} \int_{0}^{\infty}\left[Q\left(\sqrt{\frac{1+\rho}{1-\rho}} X\right)+Q\left(\sqrt{\frac{1-\rho}{1+\rho}} X\right)\right] e^{-\left(X+\sqrt{2 \gamma_{b}}\right)^{2} / 2} d x\right\}
\end{aligned}
$$

which is determined by the value of $|\rho|$. Fig. 3 shows this error probability for several values of $|\rho|$. The best performance is given when

As (23) shows, the exact error probability for noncoherent detection of pulse position modulated UWB signal is not expressed in closed-form. However, when $\left.\gamma_{b}\right\rangle 6.3(8 d B)$, it can be well approximated by the first two terms on the right side of (23), i.e., $P_{e}\left(\gamma_{b}\right) \approx Q\left(\sqrt{(1+\rho) \gamma_{b}}\right)+Q\left(\sqrt{(1-\rho) \gamma_{b}}\right)$, for $\gamma_{b}>6.3(8 d B)$

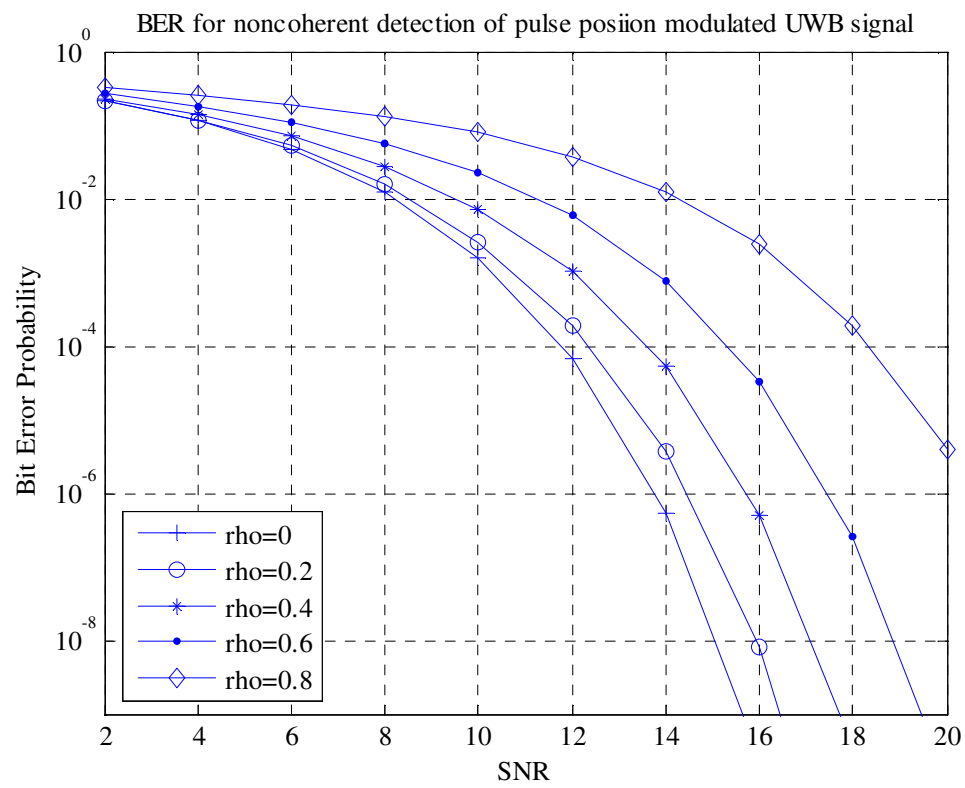

Figure 3: The error probability for noncoherent detection of pulse position modulated UWB signal corresponding to different value of $\rho$.

This approximation is illustrated in Fig. 3 for $\rho=0$ and 0.8. Because a signal-to-noise ratio of more than $8 \mathrm{~dB}$ is usually needed in practice to secure a reasonable bit error probability (less than $10^{-2}$ ), we can use (24) as a satisfactory theoretical reference for noncoherent detection of pulse position modulated UWB signal.

\section{CONCLUSION}

The exact evaluation of bit error probabilities for UWB systems with biphase modulation, pulse position modulation, and hybrid modulation in ideal AWGN channel is presented in this paper. 
Closed-form approximations of performances for non-coherent pulse position modulated and hybrid modulated systems are also given. The results provide theoretical references for UWB system performances in other non-ideal channels. It is shown that the performance of a UWB system depends not only on the modulation scheme and detection method it uses but also on the normalized auto-correlation function of the received signal impulse. This observation gives raise to the issue of optimal UWB signal design and modulation parameter selection for system performance optimization. As a design example, the optimal time shifts in pulse position modulation and hybrid modulation are determined for the widely used Gaussian monocycle UWB impulse.

\section{REFERENCES}

[1] M. Z. Win, R. A. Scholtz, and M. A. Barnes,(1997) "Ultra-wide bandwidth signal propagation for indoor wireless communications," in Proceedings of IEEE International Conference on communications, Vol. 1, pp. 56-60.

[2] M. Z. Win and R. A. Scholtz,(1998) "On the robustness of ultra-wide bandwidth signals in dense multipath environments," IEEE Communications Letters, Vol. 2, No. 2, pp. 51-53.

[3] M. Z. Win and R. A. Scholtz,(1998) "On the energy capture of ultra wide bandwidth signals in dense multipath environments," IEEE Communications Letters, Vol. 2, No. 9, pp. 245-247.

[4] F. Ramírez-Mireles, M. Z. Win and R. A. Scholtz,(1997) "Signal selection for the indoor wireless impulse radio channel," in Proceedings of IEEE Vehicular Technology Conference, pp. 2243-2247.

[5] F. Ramírez-Mireles and R. A. Scholtz,(1998) "System performance analysis of impulse radio modulation," in Proceedings of Radio and Wireless Conference, pp. 67-70.

[6] M. Z. Win and R. A. Scholtz, (1998) "Impulse radio: How it works," IEEE Communications Letters, Vol. 2, No. 2, pp. 36-38

[7] M. Z. Win and R. A. Scholtz, (2000) "Ultra-wide bandwidth time-hopping spread-spectrum impulse radio for wireless multiple-access communications," IEEE Transactions on Communications, Vol. 48, No. 4, pp. 679-691.

[8] H. Lee, B. Han, Y. Shin and S. Im,(2000) "Multipath characteristics of impulse radio channels," in Proceedings of IEEE Vehicular Technology Conference 2000 Spring, pp. 2487-2491,Tokyo.

[9] Y. Li and X. Huang,(2000) "The spectral evaluation and comparison for ultra wideband signals with different modulation schemes," in Proceedings of SCI2000, Vol. VI, pp. 277-282, Orlando.

[10] X. Huang and Y. Li, (2001) "Generating near-white ultra-wideband signals with period extended PN sequences," in Proceedings of Vehicular Technology Conference, Vol. 2, pp. 11841188, Rhodes.

[11] "Characterization of ultra-wide bandwidth wireless indoor channels: A communication-theoretic view,” IEEE J. Select. Areas Commun, vol. 20, pp. 1613-1627, Dec. 2002.

[12] M. Win and R. Scholtz,(1998) "Impulse radio: How it works," IEEE Commun. Lett., vol. 2, pp. 36-38.

[13] F. Ramirez-Mireles, (2001) "Performance of ultra wide band SSMA using time hopping and Mary PPM,” IEEE J. Select. Areas Commun., vol. 19, pp. 1186-1196.

[14] M. Win and R. Scholtz,(1997) "Energy capture vs. correlator resources in ultrawide bandwidth indoor wireless communications channels," in Proc.MILCOM, vol. 3, pp. 1277-1281.

[15] M. K. Simon, (1995) Digital Communication Techniques. Upper Saddle River, NJ: PrenticeHall.

[16] V. Somayazulu, J. Foerster, and S. Roy,( 2002) "Design challenges for very high data rate UWB systems,"in Proc. 36th Asilomar Conference on Signals, Systems and Computers, vol. 1, pp. 717-721. 
[17] Y. Ma, F. Chin, B. Kannan, and S. Pasupathy,(2002) "Acquisition performance of an ultra wideband communications system over a multiple-access fading channel," in Proc. IEEE Conf. Ultra Wideband Systems and Technologies, pp. 99-103.

[18] W. Lovelace and J. Townsend,(2002) "The effects of timing jitter and tracking on the performance of impulse radio,” IEEE J. Select. Areas Commun., vol. 20, pp. 1646-1651.

[19] J. Choi and W. Stark,(2002) "Performance analysis of RAKE receivers for ultra-wideband communications with PPM and OOK in multipath channels," in Proc. ICC, vol. 3, pp. 19691973.

[20] M. Z. Win, G. Chrisikos, and N. R. Sollenberger, (2000) "Performance of rake reception in dense multipath channels: Implications of spreading bandwidth and selection diversity order," IEEE J. Select. Areas Commun., vol. 18, pp. 1516-1525.

[21] J. G. Proakis, Digital Communications, McGraw-Hill, Inc., Third Edition, 1995.

\section{Authors}

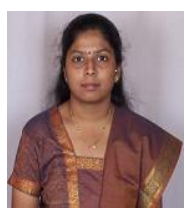

A. P. Adsul is working as a Lecturer in Vishwakarma Institute of Technology, Pune-37. She received bachelor's degree in Electronics in 1995, ME in Information Technology in 2006. Currently she is pursuing Ph.D. in NMIMS University, Mumbai, India under the guidance of Dr. S. K. Bodhe

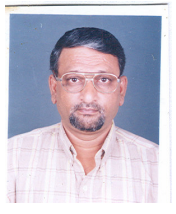

S. K. Bodhe born in Pune, India on Sept. $16^{\text {th }} 1959$. He received bachelor's degree in $\mathrm{E} \& \mathrm{TC}$ in 1983, M.E. in 1987 and Ph. D. in 1997 from Shivaji University, Kolhapur. He started his career as a R\&D engineer turned teacher after 3 years. He became Assistant Professor in 1990 in Walchand College of Engineering in Sangli and Professor in 1999 in Pune Institute of Computer Technology. Currently he is working as a Principal, College of Engineering, Pandharpur, India. His area of interest is wireless networks. 